CASE REPORT

\title{
Idiopathic Large Pericardial Effusion and Cardiac Tamponade in Children with Down Syndrome

\author{
Numra Aleem ${ }^{*}$, MD, Amna Qasim ${ }^{1}, M D$ and Ashraf M Aly ${ }^{2}, M D$, PhD \\ ${ }^{1}$ Department of Pediatrics, University of Texas Medical Branch, Galveston, TX, USA \\ ${ }^{2}$ Division of Pediatric Cardiology, University of Texas Medical Branch, Galveston, TX, USA
}

*Corresponding author: Numra Abdul Aleem, MD, Department of Pediatrics, University of Texas Medical Branch, 3.23 Research Building 6, 301 University Blvd, Galveston, TX-77555-0361, USA, Tel: 409-392-3730

\begin{abstract}
Keywords
Pericardial disease, Echocardiography, Congenital heart disease
\end{abstract}

\section{Introduction}

Pericardial effusions (PE) in patients with Down syndrome (Trisomy 21) have been associated with hypothyroidism, viral infections and malignancies (particularly leukemia). The PE is usually not large enough to cause cardiac tamponade. We report two cases of idiopathic large PE with one of them progressing to develop a cardiac tamponade that required an urgent pericardiocentesis.

\section{Case 1}

A 3-year-old female with Trisomy 21 was seen at cardiology clinic for follow up of a small secundum ASD. Patient has been asymptomatic from the cardiac standpoint and the parents denied any recent fever or other acute illness. Her cardiac examination and EKG were normal. An echocardiogram (ECHO) showed a normal 4-chamber anatomy and a small size $(5-7 \mathrm{~mm})$ pericardial effusion that had progressively increased and remained stable over a 2-year course $(9-15 \mathrm{~mm}$ ) (Figure 1). Work-up to discern the etiology of the pericardial effusion showed normal $C B C$, thyroid studies, and inflammatory markers. The rheumatologic workup (C3, C4, ANA, Anti-DsDNA, Anti SMRNP, CCP, and ANCA) and viral respiratory panel (Adenovirus, Enterovirus, Rhinovirus, Influenza and para influenza virus) were all negative. The PE was deemed to be idiopathic and the patient is being managed conservatively. She remains asymptomatic and the most recent $\mathrm{ECHO}$ at 5 years of age showed a 7-9 mm PE.

\section{Case 2}

A 2.5-year-old female with Trisomy 21 who was known to have a small secundum ASD and was lost to follow-up for 2 years was seen in cardiology clinic. Parents reported upper respiratory symptoms, de-

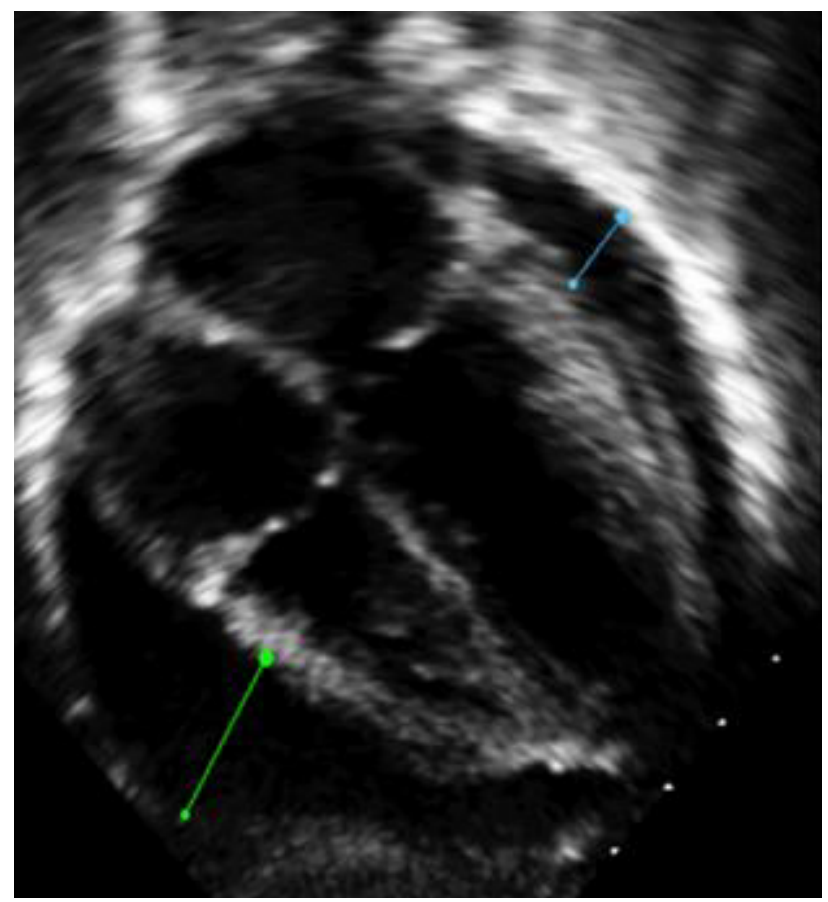

Figure 1: A large (9-15 mm) PE seen in case 1.

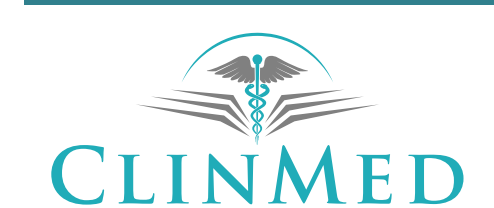

INTERNATIONAL LIBRARY
Citation: Aleem N, Qasim A, Aly AM (2020) Idiopathic Large Pericardial Effusion and Cardiac Tamponade in Children with Down Syndrome. Int J Clin Cardiol 7:165. doi.org/10.23937/2378-2951/1410165 Accepted: February 24, 2020; Published: February 26, 2020

Copyright: (c) 2020 Aleem N, et al. This is an open-access article distributed under the terms of the Creative Commons Attribution License, which permits unrestricted use, distribution, and reproduction in any medium, provided the original author and source are credited. 


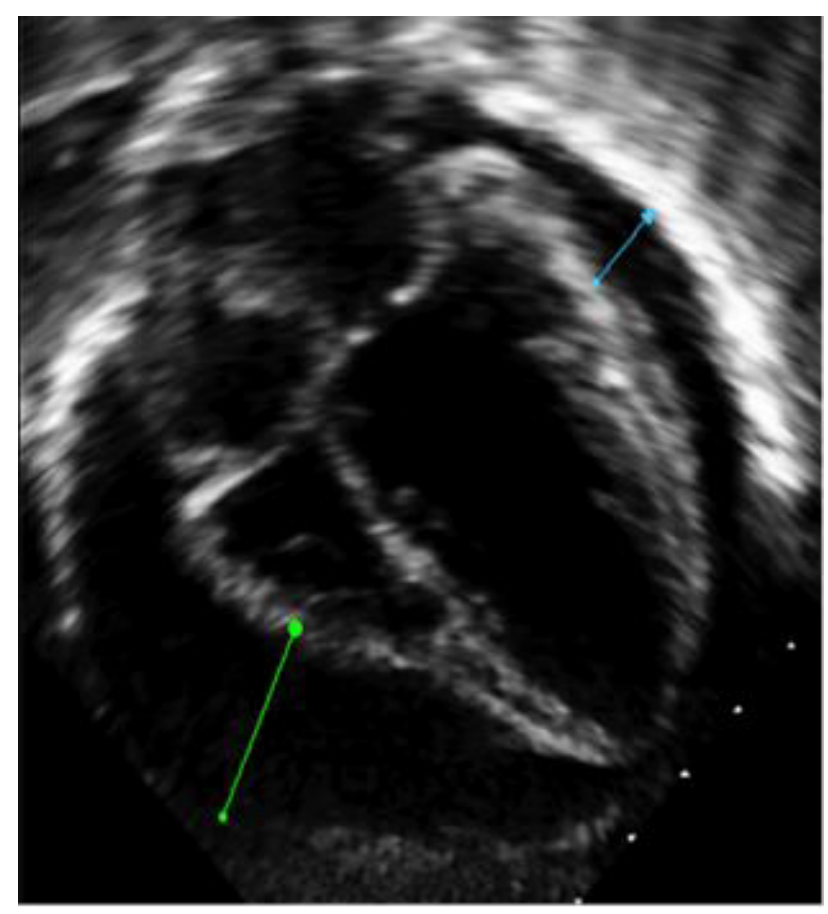

Figure 2: A large (17-22 mm) PE leading to compression of $R V$ and $R A$ resulting in cardiac tamponade physiology.

creased activity level, and shortness of breath over the few days before the visit. Physical exam was notable for grunting, subcostal retractions, and distant heart sounds. ECHO showed a large circumferential pericardial effusion (17-22 $\mathrm{mm}$ ) with a remarkable diastolic collapse of the free wall of the right atrium and right ventricle consistent with cardiac tamponade (Figure 2). Due to the severity of symptoms, the patient was transferred to the pediatric intensive care unit where an urgent ECHO guided pericardiocentesis was performed. Workup for the etiology of $\mathrm{PE}$ revealed normal thyroid studies, electrolytes, urinalysis and inflammatory markers. CBC was remarkable for severe iron deficiency. Negative microbiologic evaluation included blood cultures, pericardial fluid culture and viral respiratory PCR panel. Workup for rheumatologic etiologies was also negative. Her pericardial effusion was deemed to be idiopathic. At her 1 and 6 -month follow-up visits, the patient was asymptomatic and the ECHO showed a small pericardial effusion (5-7 $\mathrm{mm}$ ).

\section{Discussion}

Children with Trisomy 21 are at an increased risk for developing PE due to viral infections, hypothyroidism [1], celiac disease [2] and transient abnormal myelopoiesis [3]. Many viruses have been known to cause PE, either as an isolated process or part of perimyocarditis or hydrops. Recently, influenza $B$ has been reported to cause severe pericardial effusion that has responded to antiviral therapy without requiring pericardiocentesis [4]. Acquired or autoimmune thyroiditis has been reported in $15-20 \%$ of the Trisomy 21 patients [5]. The extravasation of protein due to reduced thyroid function leads to a buildup of fluid in the serous cavities including the pericardium. A similar pathophysiology is proposed to be the basis of $\mathrm{PE}$ in leukemia/myeloproliferative syndromes in which a high leukocyte and blast count leads to an exudative effusion. A myelodysplastic syndrome referred to as transient abnormal myelpoiesis (TAM) has been found in $10 \%$ of the Down syndrome infants $[6,7]$ with associated PE [3].

Isolated small to moderate size PE are often observed in patients with Down syndrome. Concolino, et al. observed patients with Trisomy 21 and PE for a 2 -year period and noted that almost $80 \%$ of them were idiopathic in origin. A few cases resolved but most persisted without any clinical symptoms and none of the cases developed a cardiac tamponade [8]. The pathophysiology behind increased pericardial fluid in Trisomy 21 patients without any comorbidity is unclear but could be supported by studies using PE as an indicator for the possible diagnosis of Trisomy 21 prenatally [9]. Idiopathic pericardial effusion may be under-reported in these patients because it is usually small and clinically insignificant and ECHO is not routinely done. The two cases we are reporting represent the extreme form of idiopathic pericardial effusion with one developing cardiac tamponade.

Certain genes on chromosome 21 have been identified to play a role in the formation of the extracellular matrix but do not cause PE [10]. A genetic link between idiopathic PE and Trisomy 21 is yet to be established [11].

\section{Conclusion}

The incidence of PE in patients with Trisomy 21 is likely to be under-reported since most patients are asymptomatic and an ECHO is not usually done. Close monitoring of patients with a moderate to large PE is needed as some may progress to develop cardiac tamponade.

\section{Acknowledgments}

We confirm that the manuscript has been read and approved by all named authors and that there are no other persons who satisfied the criteria for authorship. No additional financial or material support was required.

\section{Sources of Funding}

Department of Pediatrics, UTMB. No grants or funding required.

\section{Disclosures}

We confirm that there are no known conflicts of interest associated with this publication nor financial support for this work that could have influenced its outcome. 


\section{References}

1. González FV, Castillo L, Pi J, Ruiz E (1998) Cardiac manifestations of primary hypothyroidism. Determinant factors and treatment response. Rev Esp Cardiol 51: 893-900.

2. Riccabona M, Rossipal E (2000) Pericardial effusion in celiac disease--an incidental finding? Wien Klin Wochenschr 112: 27-31.

3. Hirashima C, Eguchi Y, Kohmura Y, Minakami H, Sato I (2000) Isolated pericardial effusion and transient abnormal myelopoiesis in a fetus with Down's syndrome. J Obstet Gynaecol Res 26: 303-306.

4. Spoto S, Valeriani E, Locorriere L, Anguissola GB, Pantano $A L$, et al. (2019) Influenza $B$ virus infection complicated by life-threatening pericarditis: $A$ unique case-report and literature review. BMC Infect Dis 19: 40.

5. Friedman DL, Kastner T, Pond WS, O'Brien DR (1989) Thyroid dysfunction in individuals with Down syndrome. Arch Intern Med 149: 1990-1993.

6. Zipursky A, Brown E, Christensen H, Sutherland R, Doyle J
(1997) Leukemia and/or myeloproliferative syndrome in neonates with Down syndrome. Semin Perinatol 21: 97-101.

7. Sacchi N (1992) Down syndrome and chromosome 21 abnormalities in leukaemia. Baillière's Clin Haematol 5: 815831.

8. Concolino D, Pascuzzi A, Pietragalla E, Lia R, Canepa S, et al. (2005) High prevalence of isolated pericardial effusion in Down syndrome. Am J Med Genet A 132A: 331-332.

9. DeVore GR (2000) Trisomy 21: 91\% detection rate using second-trimester ultrasound markers. Ultrasound Obstet Gynecol 16: 133-141.

10. Von Kaisenberg CS, Krenn V, Ludwig M, Nicolaides $\mathrm{KH}$, Brand-Saberi B (1998) Morphological classification of nuchal skin in human fetuses with trisomy 21,18 , and 13 at $12-18$ weeks and in a trisomy 16 mouse. Anat Embryol 197: 105-124.

11. Rougemont AL, Makrythanasis $P$, Finci $V$, Billieux $M H$, Epiney $M$, et al. (2010) Myeloid proliferation without GATA1 mutations in a fetus with Down syndrome presenting in utero as a pericardial effusion. Pediatr Dev Pathol 13: 423-426. 